\title{
Persistent Increase of Alcohol-Seeking Evoked by Neuropeptide S: an Effect Mediated by the Hypothalamic Hypocretin System
}

\author{
Nazzareno Cannella',3, Daina Economidou',3, Marsida Kallupi',3, Serena Stopponi', Markus Heilig', \\ Maurizio Massi' and Roberto Ciccocioppo*,' \\ 'Department of Pharmacological Sciences and Experimental Medicine, University of Camerino, Via Madonna delle Carceri, Camerino, Italy; \\ ${ }^{2}$ Laboratory of Clinical and Translational Studies, NIAAA/NIH, Bethesda, MD, USA
}

\begin{abstract}
The association of ethanol's reinforcing effects with specific environmental stimuli is thought to be a critical factor for relapse risk in alcoholism. This study examined in rats the effects of a newly deorphanized neuropeptide receptor and its cognate ligand, Neuropeptide S (NPS), on ethanol consumption and reinstatement of ethanol-seeking by environmental cues previously associated with ethanol availability. In the self-administration experiments, the stable response rates observed for ethanol reinforcement were not modified by intracerebroventricular (ICV) injection of NPS ( 1.0 and $2.0 \mathrm{nmol}$ per rat). In the reinstatement experiments, ethanol-associated cues induced robust rates of ethanol seeking, which were highly resistant to extinction over repeated sessions of reinstatement testing. ICV NPS treatment (I.0, 2.0 and $4.0 \mathrm{nmol}$ per rat) resulted in a significant increase of ethanol seeking elicited by ethanol-associated cues. In contrast, NPS did not affect the reinstatement of responding to water-paired stimuli. Site-specific NPS injection ( 0.1 and $0.5 \mathrm{nmol}$ per rat) into the lateral hypothalamus also reinstated extinguished responding to ethanol. This effect was selectively blocked by pre-treatment with the hypocretin-I/orexin-A antagonist SB-334867 (10 mg/ $/ \mathrm{kg}$, i.p.). At the dose tested, SB-334867 did not modify alcohol reinstatement per se. These results provide the first demonstration that activation of NPS receptors in the LH intensifies relapse to ethanol-seeking elicited by environmental conditioning factors. This effect is selective, and is mediated by activation of LH hypocretin neurones. Based on the present findings, we also predict that antagonism at NPS receptors could represent a novel pharmacological approach to alcohol relapse treatment.

Neuropsychopharmacology (2009) 34, 2125-2I34; do::I0.1038/npp.2009.37; published online 25 March 2009
\end{abstract}

Keywords: neuropeptide S; alcohol; addiction; relapse; hypocretin-I/orexinA; rat

\section{INTRODUCTION}

Recently, a new neuropeptide system, neuropeptide $S$ (NPS), has been deorphanized (Xu et al, 2004). NPS binds to a Gs/Gq protein-coupled receptor previously identified as GPR154 and now referred to as NPSR. Brain in situ hybridization revealed that the NPS peptide is expressed principally in a group of previously undescribed neurons located between the locus ceruleus (LC), the barrington, and the parabrachial nuclei. In the area between the LC and the barrington nucleus, NPS mRNA is predominantly expressed in glutamatergic cells, whereas in the parabrachial area, its expression overlaps that of corticotrophin releasing factor

*Correspondence: Dr R Ciccocioppo, Department of Pharmacological Sciences and Experimental Medicine, University of Camerino, Via Madonna delle Carceri, 62032 Camerino, Italy, Tel: + 390737403300 , Fax: + 390737 403325, E-mail: roberto.ciccocioppo@unicam.it

${ }^{3}$ These authors contributed equally to the study

Received 28 November 2008; revised 19 February 2009; accepted 19 February 2009 positive neurones (Xu et al, 2004, 2007). NPS receptor mRNA is expressed throughout the central nervous system, with the highest concentration found in olfactory structures, in the amygdaloid complex, in the subiculum, in the lateral, dorsomedial and ventromedial hypothalamus, and in the paraventricular thalamic nucleus (Xu et al, 2004, 2007). Activation of NPSR by intracerebroventricular (ICV) injection of NPS evokes a unique pattern of responses, including stimulation of locomotor activity, increased arousal and suppression of all stages of sleep, but also decreased experimental anxiety (Reinscheid et al, 2005; Xu et al, 2004). Its profile of action as a factor of arousal was confirmed at an endocrinological level by the observation that ICV injection of NPS also stimulates activity in the hypothalamus-hypophysis-adrenal axis (HPA), leading to increased plasma levels of ACTH and corticosterone (Smith et al, 2006).

The present study sought to determine whether the NPS system might have a role in the regulation of alcohol-related behaviors. Two main reasons prompted us to hypothesize 
the involvement of the NPS system in alcohol-addiction. First, NPSR mRNA is abundantly expressed in brain regions (ie, amygdala, hippocampus, and lateral hypothalamus) that are known to play a significant role in the regulation of alcohol drinking and reinstatement of alcohol seeking (Heilig and Koob, 2007; Le and Shaham, 2002). Second, the arousing effects of NPS together with its activation of the HPA axis system may establish a condition resembling the pathophysiological state associated with excessive alcohol use or that preceding relapse (Adinoff et al, 2005; Cleck and Blendy, 2008).

To address this hypothesis, we first investigated how ICV NPS injection affects ethanol self-administration and the relapse to alcohol-seeking induced by conditioned environmental stimuli. We found that NPS potently increases conditioned reinstatement of ethanol-seeking without affecting alcohol intake. Furthermore, NPS shows several similarities with the Hypocretin-1/Orexin-A (Hcrt-1/Ox-A) system; for example both have effects promoting arousal and wakefulness (de Lecea et al, 1998; de Lecea and Sutcliffe, 2005; Hagan et al, 1999; Sakurai et al, 1998). Moreover, it was recently reported that ICV injection of NPS induces Fos expression in the lateral hypothalamus (LH) hypocretinergic neurons (Niimi, 2006). Based on these findings, and in light of the significant role of the Hcrt-1/ Ox-A system in the regulation of relapse to drug seeking (Boutrel et al, 2005; Harris et al, 2005; Lawrence et al, 2006), we used the Hcrt-1/Ox-A receptor selective antagonist SB334867 to investigate the possibility that the NPS and the hypocretin systems interact in the LH.

\section{MATERIALS AND METHODS}

\begin{abstract}
Animals
Male Wistar rats (Charles River) weighing $175-225 \mathrm{~g}$ at the beginning of the experiments were used. Pairs of rats were housed in a room with artificial $12: 12 \mathrm{~h}$ light/dark cycle (lights off at 0800 hours), at constant temperature $\left(20-22^{\circ} \mathrm{C}\right)$ and humidity $(45-55 \%)$. All training and experimental sessions were conducted once a day during the nocturnal phase of the light/dark cycle, and each experiment used independent groups of rats. All procedures adhered to the European Community Council Directive and the National Institutes of Health Guidelines for Care and Use of Laboratory Animals.
\end{abstract}

\section{Intracranial Surgery}

For intracranial surgery, animals were anesthetized by intramuscular injection of $100-150 \mu \mathrm{l}$ of a solution containing tiletamine hydrochloride $(58.17 \mathrm{mg} / \mathrm{ml})$ and zolazepam hydrochloride $(7.5 \mathrm{mg} / \mathrm{ml})$. A guide cannula $(0.65 \mathrm{~mm}$ outside diameter) for drug injections was stereotaxically implanted and cemented to the skull. For cannula placements, the coordinates in millimeters with reference to bregma were as follows: ICV, anteroposterior (AP), -1.0; lateral (L), -1.8; ventral (V), 2.0; $\mathrm{LH}, \mathrm{AP}-1.8$; $\mathrm{L} \pm 1.8 ; \mathrm{V}-7.5$. For the $\mathrm{LH}$, cannulae were implanted bilaterally.

\section{Drug Injection and Histological Analysis}

The selective non-peptide hypocretin-1/orexin-A receptor antagonist SB334867, purchased from Tocris (Bristol, UK), was dissolved in $10 \%(\mathrm{v} / \mathrm{v})$ (2-hydroxypropyl)- $\beta$-cyclodextrin (Sigma-Aldrich, Milano, Italy), 1\% (v/v) DMSO (SigmaAldrich, Milano, Italy), diluted with sterile water and administered i.p. in a volume of $1 \mathrm{ml} / \mathrm{kg}$.

NPS, a generous gift of Dr R Guerrini, University of Ferrara, Italy, was dissolved in sterile isotonic saline and injected ICV in a volume of $1 \mu \mathrm{l}$ per rat by means of a stainless-steel injector $2.5 \mathrm{~mm}$ longer than the guide cannula, so that its tip protruded into the ventricle. For an intra-LH experiment, rats received $1.0 \mu \mathrm{l}$ of solution injected at a volume of $0.5 \mu \mathrm{l}$ per each cannula through an injector $1.6 \mathrm{~mm}$ longer than the guide cannula.

At the end of each experiment, rats were killed by $\mathrm{CO}_{2}$ inhalation, and perfused transcardially with isotonic saline followed by $3 \%$ formalin. Brains were removed, stored in formalin and subsequently sectioned into $30 \mu \mathrm{m}$ coronal sections using a cryostat. After staining with cresyl violet, sections were examined under a microscope for the location of the injector tip placement. An expert observer blind to treatment conditions and behavioral data was used for histological verification. Only animals with correct cannulae placements were used for a statistical analysis.

\section{Operant Training and Ethanol Self-Administration}

Operant self-administration chambers (Med Associates) equipped with a drinking reservoir (volume capacity: $0.30 \mathrm{ml}$ ) and two retractable levers were used. Auditory and visual stimuli were presented through a speaker and a light located on the front panel. Rats were trained to selfadminister $10 \%$ alcohol (v/v) in 30-min daily sessions on a fixed-ratio 1 schedule of reinforcement, in which each response resulted in delivery of $0.1 \mathrm{ml}$ of fluid, as described earlier (Economidou et al, 2006). In all the experiments, the number of operant responses at both the active and the inactive levers was recorded.

\section{Conditioned Reinstatement of Alcohol-Seeking Behavior}

This experiment consisted of three phases:

Conditioning phase. In $30 \mathrm{~min}$ daily sessions, animals were trained to discriminate between $10 \%$ alcohol and water. Beginning with self-administration training at the $10 \%$ alcohol concentration, discriminative stimuli (SD) predictive of alcohol $v s$ water availability were presented during the alcohol and water self-administration sessions, respectively. The discriminative stimulus for alcohol was the odor of an orange extract $(S+)$, whereas water availability (ie, no reward) was signaled by an anise extract $(\mathrm{S}-$ ). The olfactory stimuli were generated by depositing six to eight drops of the respective extract into the bedding of the operant chamber. In addition, each lever-press resulting in delivery of alcohol was paired with illumination of the chamber's house light for $5 \mathrm{~s}$. The corresponding cue during water sessions was a $5 \mathrm{~s}$ tone $(70 \mathrm{~dB})$. Concurrently with the presentation of these stimuli, a $5 \mathrm{~s}$ time-out period was in 
effect, during which responses were recorded but not reinforced. The olfactory stimuli serving as $S+$ or $S-$ for alcohol availability were introduced one minute before extension of the levers and remained present throughout the $30-\mathrm{min}$. sessions. Alcohol and water sessions were conducted in random order across training days, with the constraint that all rats received a total of 10 alcohol and 10 water sessions.

Extinction phase. After the last conditioning day, rats were subjected to 30 -min extinction sessions for 15 consecutive days. During this phase, sessions began by extension of the levers without presentation of the SD. Responses at the lever activated the delivery mechanism but did not result in the delivery of liquids or the presentation of the responsecontingent cues (house light or tone).

Reinstatement testing. Reinstatement tests began the day after the last extinction session. This test lasted 30-min under conditions identical to those during the conditioning phase, except that alcohol and water were not made available. Wistar rats were tested under the $S+$ condition on day 1 and under the $S-$ condition on day 2 . Subsequently, the effect of NPS on cue-induced reinstatement was evaluated.

\section{Experiment 1: Effect of ICV NPS Treatment on Alcohol} Self-Administration

Rats $(n=8)$ were trained to self-administer $10 \%(\mathrm{v} / \mathrm{v})$ ethanol as described earlier. Following acquisition of a stable baseline of alcohol self-administration, NPS treatment began. In counterbalanced order, $5 \mathrm{~min}$ before the beginning of the self-administration session, rats were injected ICV with NPS (1.0 and $2.0 \mathrm{nmol}$ per rat) or its vehicle, respectively. Drug treatment was performed every fourth day. The first day after drug tests, rats remained in their home cages, whereas on days 2 and 3 after the test baseline ethanol self-administration was re-established.

\section{Experiment 2: Effect of ICV NPS Treatment on Cue- Induced Reinstatement of Alcohol-Seeking Behavior}

Three days after the first reinstatement test, the effect of NPS on cue-induced reinstatement of alcohol seeking was studied in a group of 8 rats. Every third day, in a counterbalanced order, animals were ICV injected with NPS $(1.0,2.0$ and $4.0 \mathrm{nmol}$ per rat) or its vehicle. Immediately after injection, rats were placed into the selfadministration chambers and tested for their operant behavior under the $\mathrm{S}+$ condition.

To evaluate the specificity of NPS $(1.0,2.0$ and $4.0 \mathrm{nmol}$ per rat) effects, another group of rats $(n=8)$ was separately tested for the effect of the peptide in response to cues predictive of water availability.

Between reinstatement tests, animals remained confined in their home cages.
Experiment 3: Effect of NPS Treatment on Cue-Induced Reinstatement of Alcohol-Seeking Behavior in a Repeated Relapse Model

In an earlier study, we have shown that in an extinction/ reinstatement paradigm, cues predictive of alcohol availability maintain their ability to elicit relapse for a prolonged period of time, and after multiple presentations (Ciccocioppo et al, 2001). Here, using this model, we investigated whether NPS is able to increase cue-induced reinstatement following multiple relapses and repeated ICV injections.

Animals $(n=16)$ were divided into two groups $(n=8 /$ group) with similar baseline number of responses during discrimination and extinction, and on day one were tested for reinstatement under the S- condition. The day after (alcohol relapse-session 1), both groups of rats were subjected to the reinstatement test under $\mathrm{S}+$ condition, but one group of rats (treated) was ICV injected with NPS $(2.0 \mathrm{nmol}$ per rat) whereas the other group of animals (control) received ICV saline. The reinstatement test was repeated every third day, and treated rats received NPS again in alcohol relapse-sessions 3 and 6, and saline in alcohol relapse-sessions 2, 4 and 5. The control group always received an ICV vehicle. Finally, two $S-$ sessions were repeated, with the treated group receiving $2 \mathrm{nmol}$ of NPS in the first session, and ICV saline in the second. The control group received ICV vehicle on both occasions. During the 3-day period that separated reinstatement tests, animals remained confined to their home cages.

\section{Experiment 4: Effect of Intra-LH Injection of NPS on Cue-Induced Reinstatement of Alcohol-Seeking Behavior}

A group of rats $(n=9)$ was bilaterally implanted with intracranial cannula aimed at the LH and the effect of sitespecific injection of NPS on cue-induced reinstatement of alcohol-seeking was investigated. The study was conducted as described in Experiment 2 with the exception that NPS $(0.03,0.1,0.5 \mathrm{nmol}$ per rat) or its vehicle were directly administered into the LH. Drug injection was given in a counterbalanced order (Latin square design) $5 \mathrm{~min}$ before the beginning of the reinstatement session.

\section{Experiment 5: Effect of SB-334867 on Cue-Induced Reinstatement of Ethanol Seeking after Intra-LH NPS Injection}

The study was performed as described in Experiment 2. Briefly, rats $(n=10)$, trained to a conditioning, extinction reinstatement procedure received in counterbalanced order SB-334867 (0.0 and $10.0 \mathrm{mg} / \mathrm{kg}$, i.p.) and, $30 \mathrm{~min}$ afterwards, an intra-LH injection of NPS $(0.5 \mathrm{nmol}$ per rat) or its vehicle. Five minutes after NPS or vehicle treatment, animals were tested for cue-induced relapse in a 30-min session. In a counterbalanced order (Latin square design), rats were subjected to all treatment conditions.

\section{Statistical Analysis}

Data were analyzed using appropriate within and between factor ANOVAs. Specifically, the results of experiment 1 
were analyzed by means of a one-factor (treatment) ANOVA for repeated measures. In experiments 2, 3, 4 and 5 , discrimination was analyzed by means of a two-factor ANOVA with one factor within (time) and one factor between (self-administration condition). Reinstatement and drug effects were analyzed separately by means of a one factor (reinstatement, or doses) within subject ANOVA. Analysis of variance was followed by the Newman-Keuls test when appropriate. Statistical significance was set at $p<0.05$.

\section{RESULTS}

\section{Histology}

Histological analysis revealed that the ICV cannulae were aimed at the right position in all the rats with implants. Conversely, correct cannula placement in the LH was observed in seven out of nine rats in experiment 4 and in seven out of 10 rats in experiment 5 (Figure 1). Rats with incorrect cannula placement were not considered in statistical analysis. Interestingly, those rats did not respond to NPS treatment.

\section{Experiment 1: Effect of ICV NPS Treatment on Alcohol Self-Administration}

Rats rapidly acquired stable ethanol self-administration. Treatment with ICV NPS did not significantly modify operant responding for ethanol $\left(\mathrm{F}_{(2,7)}=0.158 ; p>0.05\right)$ (Figure 2a).

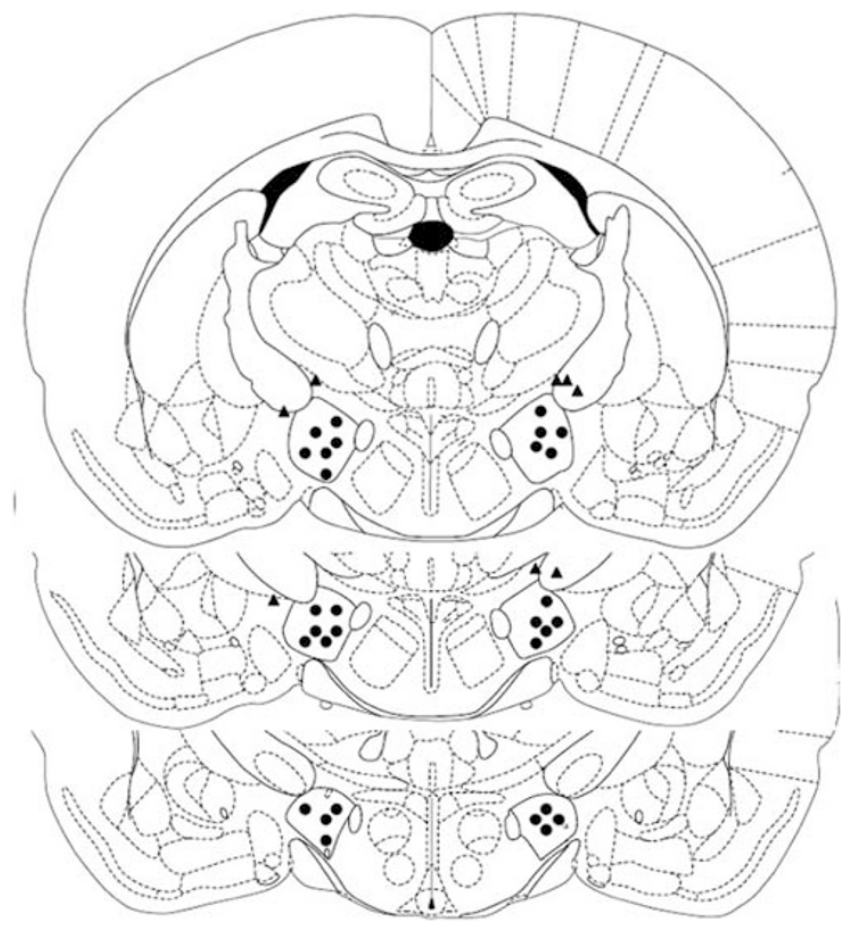

Figure I Histological reconstruction showing correct (filled circles) and incorrect (filled triangles) injections in the LH. Data presented in the figure are representative of the whole samples used in the study, and indicative of the criteria used for identification of correct cannula sites. The drawing is from the Paxinos and Watson atlas (1986).
Responses at the inactive lever were almost absent throughout the experiment and were not affected by NPS treatment $\left(\mathrm{F}_{(2,7)}=0.811 ; p>0.05\right)$ (Figure $2 \mathrm{~b}$ ).

\section{Experiment 2: Effect of ICV NPS Treatment on Cue-Induced Reinstatement of Alcohol-Seeking Behavior}

ANOVA revealed a significant overall effect of conditioning $\left(\mathrm{F}_{(1,14)}=287.98 ; p<0.0001\right)$ during the discrimination phase. Animals responded at a significantly higher level for ethanol than for water. Ethanol self-administration progressively increased during the discrimination period and passed from $32.37 \pm 3.24$ in the first session to $48.42 \pm 5.97$ in the last session. Conversely, water selfadministration progressively decreased and passed from $33.74 \pm 4.39$ on the first day to $24.37 \pm 2.74$ on the last day. During extinction, lever pressing progressively decreased, from $37.86 \pm 6.85$ on the first day to $6.71 \pm 1.05$ on the last extinction day. In the reinstatement test, the ANOVA showed that cues had a significant overall effect on ethanolseeking $\left(\mathrm{F}_{(2,7)}=16.655 ; p<0.001\right)$ (Figure 3a). A more detailed analysis showed a robust reinstatement of responding under the $\mathrm{S}+(p<0.001)$, but not under the $\mathrm{S}-$ condition. Reinstatement testing did not affect inactive lever responses $\left(\mathrm{F}_{(2,7)}=1.109 \quad p>0.05\right)$ (Figure 3b). Reinstatement of ethanol-seeking was significantly increased by pre-treatment with NPS $\left(\mathrm{F}_{(3,7)}=3.832\right.$; $p<0.05)$. Post hoc comparisons showed a significant effect

a

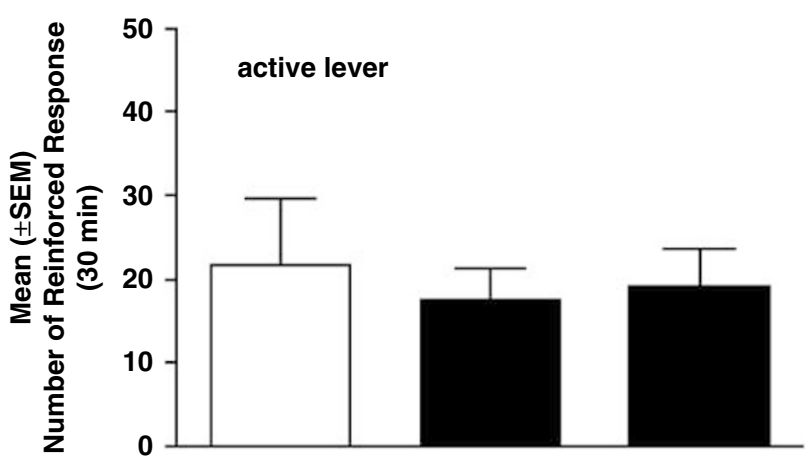

b

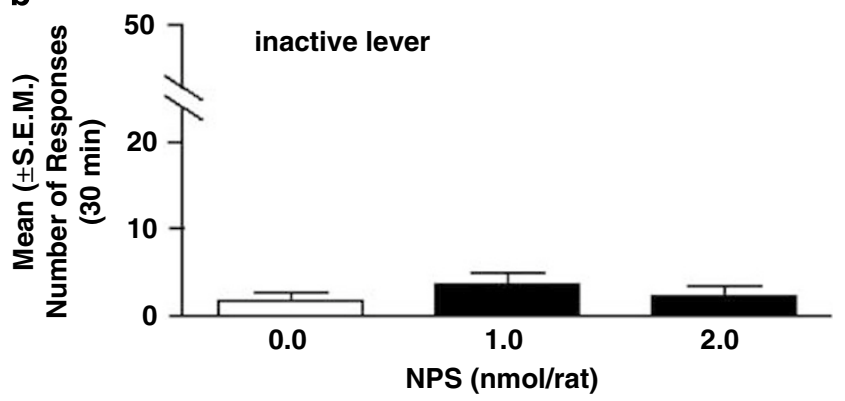

Figure 2 Effect of ICV treatment with NPS ( 1.0 and $2.0 \mathrm{nmol}$ per rat) or its vehicle $(0.0)$ on alcohol self-administration in Wistar rats $(N=8)$ under an FR-I schedule of reinforcement; (a) active lever, (b) inactive lever. Values represent the mean ( \pm SEM) number of reinforced responses at the active lever or nonreinforced responses at the inactive lever. Difference from vehicle-treated rats (controls) was not significant. 
a Discrimin. Reinstatement

Phase Test
ICV

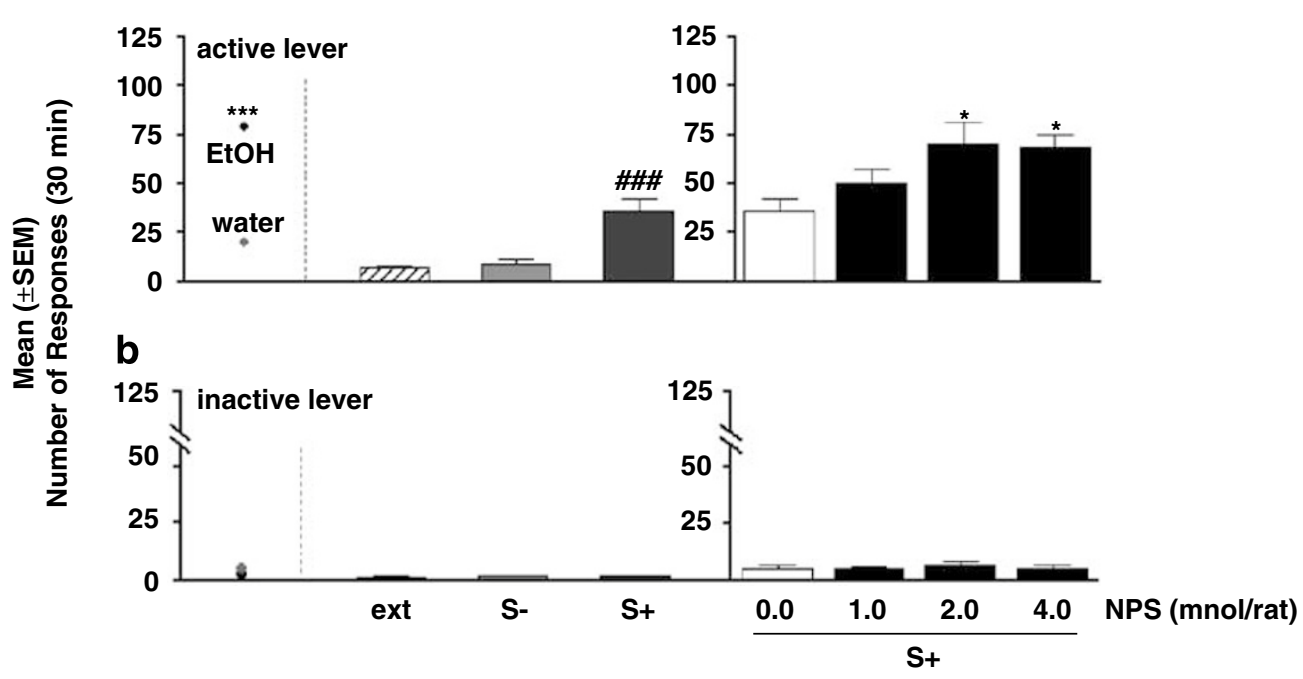

Figure 3 Reinstatement, responses in rats $(N=8)$ exposed to $S+$ and $S$ - conditions (in the absence of reward delivery) compared with the mean number of lever presses of the last 3 days of extinction (EXT). Effect of ICV injection of NPS (I.0, 2.0 and 4.0 nmol per rat) or its vehicle (0.0) on cueinduced reinstatement of alcohol-seeking $(S+)$. (a) Mean ( \pm SEM) number of responses at the alcohol active lever; (b) Mean ( \pm SEM) number of responses at the alcohol inactive lever. Black circle $(\mathrm{EtOH})$ and gray circle (water) represent the last ethanol and water discrimination sessions, respectively. Difference between alcohol and water response ${ }^{*} * * * 0.001$. Difference from extinction, ${ }^{\# \# \#} p<0.001$. Difference from vehicle-treated rats (controls), ${ }^{*} p<0.05$.

of NPS at the doses of 2.0 and $4.0 \mathrm{nmol}$ per rat $(p<0.05)$ (Figure 3a). The number of responses at the inactive lever was very low and was not affected by NPS treatment $\left(\mathrm{F}_{(3,7)}=0.653 ; p>0.05\right)$ (Figure $3 \mathrm{~b}$ ).

In the group of rats used to test the effect of NPS on cues predictive of water availability, ANOVA showed a significant overall effect of conditioning $\left(\mathrm{F}_{(1,14)}=9.55 ; p<0.01\right)$. As for the previous group, the number of ethanol responses exceeded that of water responses. Ethanol self-administration progressively increased during the discrimination period and passed from $40.87 \pm 3.65$ of the first session to $51.25 \pm 9.22$ of the last session. Conversely, water selfadministration progressively decreased and passed from $35.00 \pm 5.06$ on the first day to $22.25 \pm 7.31$ on the last day. During extinction, lever pressing progressively decreased, from $44.62 \pm 5.02$ on the first day to $9.08 \pm 0.91$ on the last extinction day. In the reinstatement test, the ANOVA showed that cues had a significant overall effect on ethanolseeking $\left(\mathrm{F}_{(2,7)}=21.954 ; p<0.0001\right)$ (Figure $\left.4 \mathrm{a}\right)$. The Newman-Keuls test demonstrated a significant reinstatement of ethanol-seeking under the $\mathrm{S}+$ stimulus condition $(p<0.001)$ but not under the $\mathrm{S}-$ one. NPS treatment did not affect lever response under $S-$ condition $\left(\mathrm{F}_{(3,7)}=0.387\right.$; $p>0.05$ ) (Figure 4a).

The number of responses at the inactive lever was very low and was not affected by NPS treatment $\left(\mathrm{F}_{(3,7)}=1.305\right.$; $p>0.05$ ) (Figure $4 \mathrm{~b}$ ).

\section{Experiment 3: Effect of NPS Treatment on Cue-Induced Reinstatement of Alcohol-Seeking Behavior in a Repeated Relapse Model}

ANOVA revealed a significant overall effect of conditioning $\left(\mathrm{F}_{(1,30)}=702.13 ; p<0.0001\right)$. Animals responded at a significantly higher level for the drug than for water. Ethanol self-administration progressively increased during the discrimination period and passed from $29.12 \pm 2.43$ for the first session to $45.44 \pm 6.01$ for the last session. Conversely, water self-administration progressively decreased and passed from $32.39 \pm 2.43$ on the first day to $17.31 \pm 4.89$ on the last day. During extinction, lever pressing progressively decreased from $37.94 \pm 5.90$ on the first day to $6.98 \pm 0.92$ for the last extinction session. At this point, animals were divided into two groups with similar levels of extinction; one was treated with NPS and one with vehicle. ANOVA revealed a significant overall effect of reinstatement $\left(\mathrm{F}_{(9,126)}=15.51 ; p<0.0001\right)$ and a significant difference between groups $\left(\mathrm{F}_{(1,126)}=11.45 ; p<0.01\right)$. More detailed analysis showed a significant reinstatement of responding in both vehicle and NPS-treated groups in all $\mathrm{S}+$ sessions (Figure 5a). Interestingly, post hoc tests identified a significant difference between NPS and vehicle control groups; on every occasion NPS was given prior to reinstatement of responding under $\mathrm{S}+$ condition (specifically, on days 1 and $3 p<0.05$; on day $6 p<0.001$ ). When NPS was given under $\mathrm{S}-$ condition, no difference between treated and controls was observed. (Figure 5a).

Responses at the inactive lever were almost absent throughout all experimental phases $\left(\mathrm{F}_{(9,126)}=1.48\right.$; $p>0.05)$ and were not affected by NPS treatment $\left(\mathrm{F}_{(1,126)}=0.18 ; p>0.05\right)$ (Figure $\left.5 \mathrm{~b}\right)$.

\section{Experiment 4: Effect of Intra-LH Injection of NPS on Cue-Induced Reinstatement of Alcohol-Seeking Behavior}

Following histological examination, seven rats were used for statistical analysis. ANOVA revealed a significant overall effect of conditioning $\left(\mathrm{F}_{(1,12)}=50.86 ; p<0.0001\right)$. Ethanol self-administration progressively increased during the discrimination period and passed from $35.14 \pm 6.62$ for the first session to $45.14 \pm 5.74$ for the last session. Conversely, 


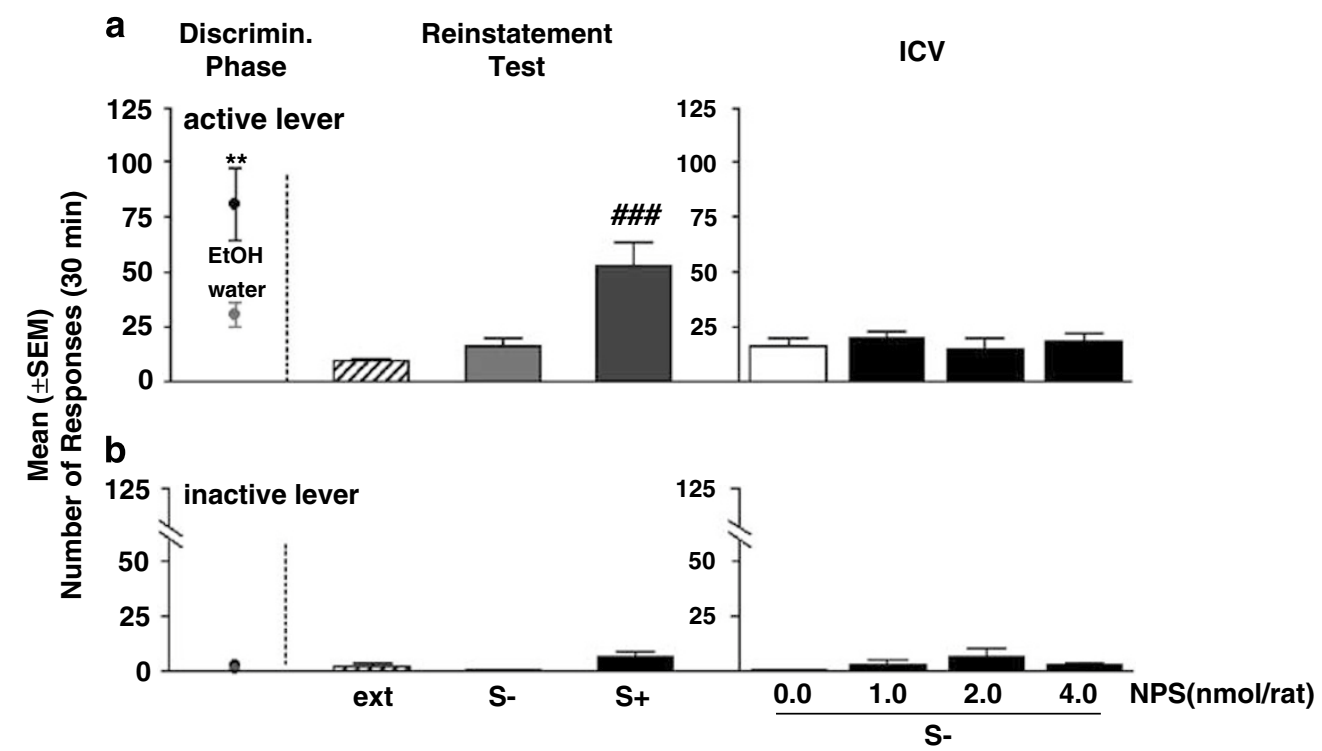

Figure 4 Reinstatement, responses in rats $(N=8)$ exposed to $S+$ and $S$ - conditions (in the absence of reward delivery) compared with the mean number of lever presses of the last 3 days of extinction (EXT). Effect of ICV injection of NPS (I.0, 2.0 and 4.0 nmol per rat) or its vehicle (0.0) on responding associated to cues predictive of water (S-) availability. (a) Mean ( \pm SEM) number of responses at the active lever; (b) Mean ( \pm SEM) number of responses at the inactive lever. Black circle $(\mathrm{EtOH})$ and gray circle (water) represent the last ethanol and water discrimination sessions, respectively. Difference between alcohol and water response $* * x<0.01$. Difference from extinction, \#\#\# $p<0.001$. Difference from vehicle-treated rats (controls) was not significant.

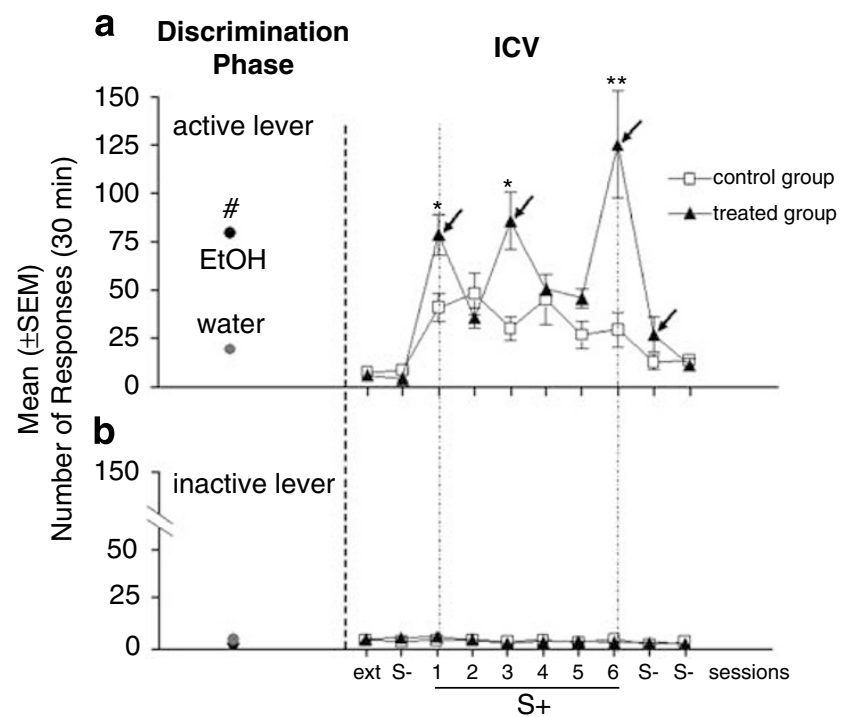

Figure 5 Responses during the repeated reinstatement test phase. One group of rats. $(N=8)$ was injected ICV with $2 \mathrm{nmol}$ per rat of NPS (dark arrow) or vehicle. Another group (control) $(N=8)$ was always injected with vehicle. Following the first two reinstatement tests, run on two consecutive days, rats were tested every third day. Exposure to the $\mathrm{S}+$ condition in the absence of further ethanol availability consistently produced a significant response-reinstatement. Responding under the Sstimulus conditions always remained at extinction (EXT) level. (a) Mean $( \pm$ SEM) number of responses at the alcohol active lever; (b) Mean ( \pm SEM) number of responses at the alcohol inactive lever. Black circle $(\mathrm{EtOH})$ and gray circle (water) represent the last ethanol and water discrimination sessions, respectively. Difference between alcohol and water response ${ }^{\#} p<0.01$. Difference from vehicle-treated rats (controls), $* p<0.05$, *** $p<0.01$.

water self-administration progressively decreased and passed from $22.71 \pm 3.34$ on the first day to $15.42 \pm 4.35$ on the last day. Animals responded at a significantly higher level for ethanol than for water $(p<0.001)$ whereas no differences were found in inactive lever response. During extinction, lever pressing progressively decreased from $41.43 \pm 7.62$ on the first day to $6.14 \pm 3.03$ on the last extinction day. In the reinstatement test, the ANOVA showed that cues had a significant overall effect on ethanolseeking $\left(\mathrm{F}_{(2,6)}=12.09 ; p<0.01\right)$ (Figure 6a). A robust reinstatement of responding was observed under the $\mathrm{S}+$ $(p<0.001)$, but not under the $S-$ condition. Reinstatement testing did not affect inactive lever response $\left(\mathrm{F}_{(2,6)}=0.48\right.$; $p>0.05$ ) (Figure 6b). Reinstatement of ethanol-seeking behavior was significantly increased by pre-treatment with NPS $\left(\mathrm{F}_{(3,6)}=8.58 ; p<0.001\right)$. Post hoc comparisons showed a significant effect of NPS at the dose of $0.5 \mathrm{nmol}$ per rat $(p<0.01)$ (Figure 6a). The $p$ value for the effect of the $0.1 \mathrm{nmol}$ dose was 0.053 . The number of responses at the inactive lever was very low and was not affected by NPS treatment $\left(\mathrm{F}_{(2,6)}=0.48 ; p>0.05\right)$ (Figure $\left.6 \mathrm{~b}\right)$. The two subjects with incorrect cannula placement showed an increase of active lever pressing in the reinstatement test (NPS vehicle; $26 \pm 12$ ). NPS failed to show its typical increase of responding (NPS 0.1 and $0.5 \mathrm{nmol}$ are $14 \pm 3.0$ and $22 \pm 5.0$, respectively), as observed in the rest of the group in which cannulae reached the $\mathrm{LH}$.

\section{Experiment 5: Effect of SB-334867 on Cue-Induced Reinstatement of Ethanol-Seeking after Intra-LH NPS Injection}

Following histological examination, seven rats were used for statistical analysis. The ANOVA showed a significant overall effect of conditioning $\left(\mathrm{F}_{(1,12)}=10.12 ; p<0.01\right)$. Animals responded at a significantly higher level for alcohol than for water $(p<0.01)$ whereas inactive lever response was very low and not subjected to conditioning. Ethanol 


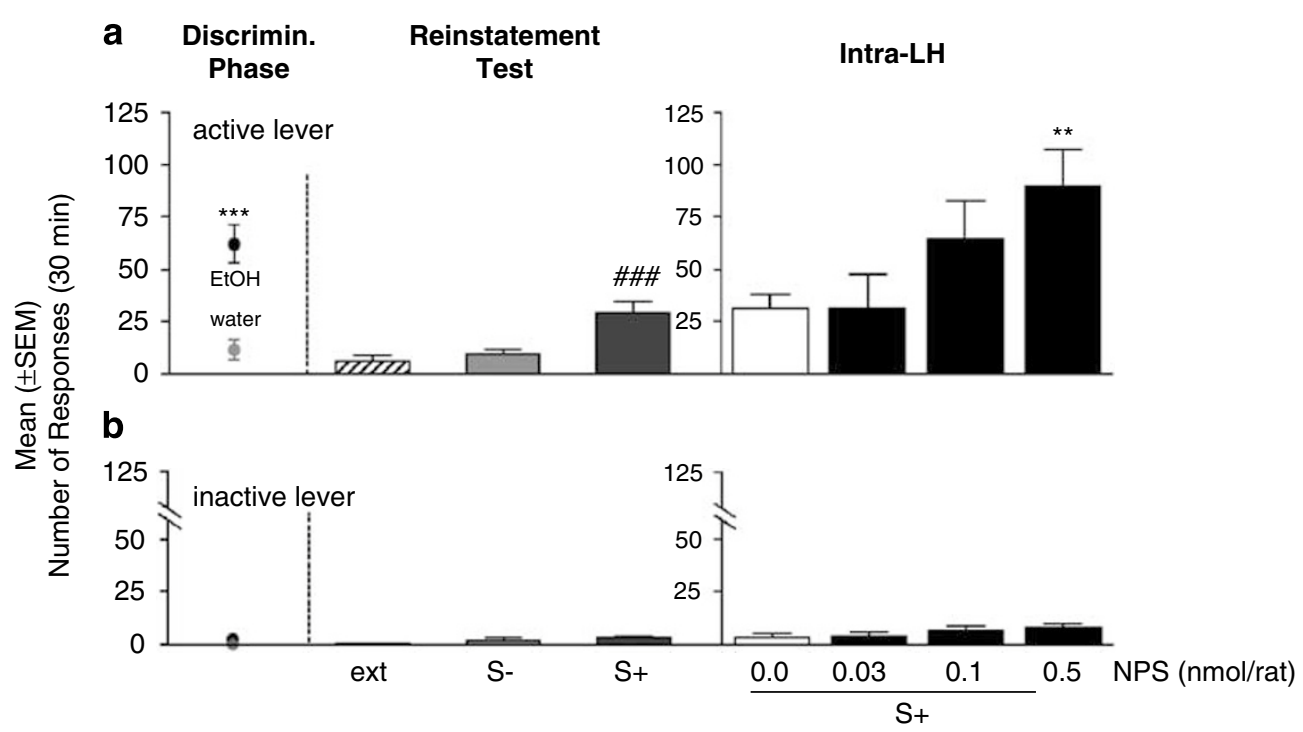

Figure 6 Effect of intra LH bilateral injection of NPS $(0.03,0.1$ and $0.5 \mathrm{nmol}$ per rat) or its vehicle $(0.0)$ on cue-induced reinstatement of alcohol-seeking in Wistar rats $(N=7)$. Reinstatement, responses in rats exposed to $S+$ and $S$ - conditions (in the absence of alcohol or saline) in comparison with the mean number of lever presses of the last 3 days of extinction (EXT). Mean ( \pm SEM) number of responses at the: (a) Mean ( \pm SEM) number of responses at the active lever. (b) Mean $( \pm$ SEM) number of responses at the inactive lever. Black circle $(E t O H)$ and gray circle (water) represent the last ethanol and water discrimination sessions, respectively. Difference between alcohol and water response $* * * *<0.001$. Difference from extinction, $\# \# \#$ p $<0.001$. Difference from vehicle-treated rats (controls), $* *$ $p<0.01$

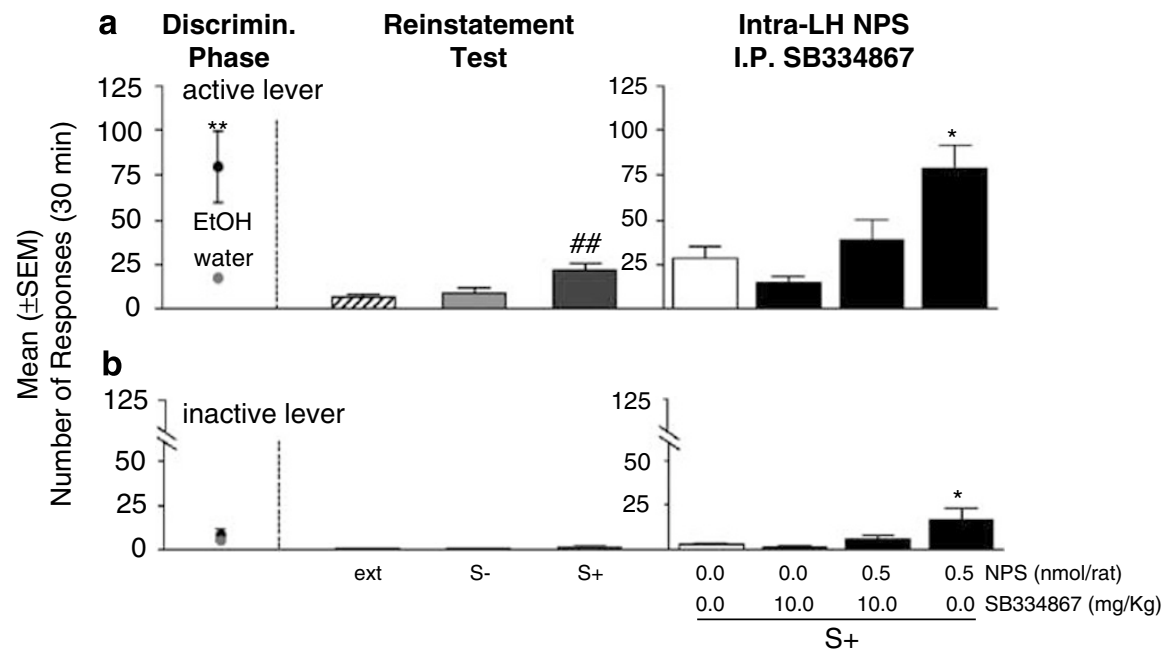

Figure 7 Effect of pre-treatment with the Hcr-A/Ox-I antagonist SB-334867 (I $0 \mathrm{mg} / \mathrm{kg}$, i.p.) or its vehicle (0.0) on intra-LH injection of NPS (0.5 nmol per rat) or its vehicle (0.0) on cue-induced reinstatement of alcohol-seeking in Wistar rats $(N=7)$. Reinstatement, responses in rats exposed to $S+$ and $S-$ conditions (in the absence of alcohol or saline) compared with the mean number of lever presses of the last 3 days of extinction (EXT). (a) Mean ( \pm SEM) number of responses at the active lever. (b) Mean ( \pm SEM) number of responses at the inactive lever. Black circle (EtOH) and gray circle (water) represent the last ethanol and water discrimination sessions, respectively. Difference between alcohol and water response $* * p<0.01$. Difference from extinction, $\# \#<0.01$. Difference from vehicle-treated rats (controls), $* p<0.05$.

self-administration progressively increased during the discrimination period and passed from $37.14 \pm 7.25$ for the first session to $45.42 \pm 7.36$ for the last session. Conversely, water self-administration progressively decreased and passed from $26.28 \pm 7.10$ on the first day to $11.32 \pm 1.57$ on the last day. During extinction, lever pressing progressively decreased from $33.14 \pm 4.66$ on the first day to $6.71 \pm 1.92$ on the last extinction day. The number of responses on the inactive lever remained very low through- out the extinction. In the reinstatement test, the ANOVA showed that cues had a significant overall effect on ethanolseeking $\left(\mathrm{F}_{(2,6)}=11.18 ; p<0.01\right)$ (Figure $\left.7 \mathrm{a}\right)$. A more detailed analysis showed a robust reinstatement of responding under the $\mathrm{S}+(p<0.01)$, but not under the $\mathrm{S}-$ condition. Reinstatement of ethanol-seeking behavior showed a significant overall effect of treatment $\left(\mathrm{F}_{(3,6)}=9.85\right.$; $p<0.001)$. Post hoc comparisons showed a significant effect after NPS injection $(p<0.05)$ that was completely prevented 
by treatment with $10 \mathrm{mg} / \mathrm{kg}$ of SB-334867. At the dose tested in our experiments, the Hcrt-1/Ox-A receptor antagonist alone did not modify cue-induced reinstatement, which remained at the same level as that of vehicle-treated rats, but significantly higher compared with extinction (Figure 7a). Analysis of inactive lever responding revealed that NPS treatment had a marginal but significant effect on the inactive lever $\left(\mathrm{F}_{(3,6)}=3.90 ; p<0.05\right)$. Post hoc analysis (Figure $7 \mathrm{~b})$ revealed a significant $(p<0.05)$ increase of responding at the highest NPS dose. Because of the effects of NPS on inactive lever responding, which is a potential measure of general (non-directed) activity and response generalization, data were reanalyzed using change-scores (active lever minus inactive lever presses). This changescore analysis replicated that of active lever responding $\left(\mathrm{F}_{(3,6)}=2.35 ; p<0.001\right)$ for the treatment, suggesting that inactive lever responding cannot account for the results obtained for active lever responding (Cippitelli et al, 2008; Le et al, 2005). The three subjects with incorrect cannula placement showed increased active lever pressing in the reinstatement test (NPS vehicle; 19.66 \pm 3.28 ). However, contrary to the rats with appropriate cannula placement, NPS $0.5 \mathrm{nmol}$ failed to potentiate the effect of cues $(24.33 \pm 8.65)$.

\section{DISCUSSION}

Our results show that NPS given ICV does not modify ongoing ethanol self-administration in rats, indicating that motivational circuitry subserving ethanol reinforcement is not directly affected by activation of NPSR. This finding is consistent with recent data obtained in our laboratory showing that this peptide does not alter intravenous cocaine self-administration (Ciccocioppo et al, 2007). Our finding is in contrast with recent data by Badia-Elder et al (2008) showing that ICV NPS injection significantly reduces ethanol drinking in genetically selected alcohol preferring $\mathrm{P}$ rats. Several factors may account for this difference. In their study, Badia-Elder et al (2008) tested the effect of NPS on home cage voluntary ethanol drinking whereas here we studied peptide effects under operant conditions. Operant selfadministration is thought to more directly gauge motivation to obtain the reinforcer, whereas home cage drinking can also be controlled by taste, calories and other factors not directly related to pharmacological reinforcement. Furthermore, the $\mathrm{P}$ rats show elevated anxiogenic-like behavior and it has been hypothesized that their excessive ethanol drinking is in part driven by ethanol's negatively reinforcing properties (ie, reduction of anxiety symptoms) (Stewart et al, 1993). NPS has anxiolytic-like effects in animal models of anxiety (Jungling et al, 2008; Xu et al, 2004); hence it is possible that this peptide decreases the incentive for ethanol intake in $\mathrm{P}$ rats by partially substituting for the anxiolytic-like effects of ethanol, thus reducing the motivation to consume the drug for its negatively reinforcing properties. Lastly, it cannot be excluded that genetic variants that directly affect the function of the NPS system have co-segregated and become fixed during the selection of the $\mathrm{P}$ line.

Our reinstatement results confirm that environmental stimuli previously associated with and predictive of ethanol availability reliably reinstate extinguished responding at a previously ethanol-paired lever (Ciccocioppo et al, 2001; Katner et al, 1999). The behavioral effects of the ethanolassociated stimuli proved highly resistant to extinction as reflected by the stability of conditioned ethanol-seeking behavior over the repeated reinstatement testing. The data suggest that throughout this time, the animals' behavior was controlled selectively by the ethanol-associated stimuli, because responding returned to extinction levels during intermittent tests conducted under stimulus conditions associated with non-reward (water). Of particular interest is the observation that ICV NPS treatment leads to a significant increase of ethanol seeking elicited by cues predictive of alcohol availability. This effect was specific for ethanol, because NPS did not affect reinstatement of responding associated with presentation of water-paired stimuli. This finding, together with data showing that ICV NPS does not change operant responding at the inactive lever, demonstrates that the NPS effect is not secondary to a non-specific increase of locomotor activation or general hyperarousal (Xu et al, 2004). Moreover, we observed that NPS induced a three-fold increase of relapse responding in rats subjected to repeated reinstatement testing, and saw no indication that they developed tolerance for this effect.

Previous studies showed that NPS stimulates locomotor activity and elicits anxiolytic-like responses at doses below $1.0 \mathrm{nmol}$ given ICV. Conversely, in this study, we observed a significant increase of reinstatement of alcohol-seeking at ICV doses of $2 \mathrm{nmol}$ or higher. This may be indicative of a complex pharmacological profile of NPS, the effects of which depend on the dose tested. On the other hand, several studies have shown selective NPS effects at ICV doses as high as $10 \mathrm{nmol}$ (Beck et al, 2005; Smith et al, 2006; Vitale et al, 2008).

The LH is one of the brain areas with the highest NPSR transcript expression levels. Moreover, the $\mathrm{LH}$ is traditionally implicated in reward and motivation. We therefore evaluated the effect of NPS microinjections directly into this area, and found that intra- $\mathrm{LH}$ administration dramatically reinstated extinguished ethanol responding. This finding suggests that the LH is an important brain site of action for NPS effects on alcohol relapse. On the other hand, it is unlikely that drug diffusion from the LH might have contributed to the effect of NPS. In fact, we found that following intra-LH injection, peptide effects were marginally significant at a dose 20 times lower, and significant at a dose eight times lower than the dose showing an effect after ICV administration. In addition, we observed that in the five rats that received off-target (dorsal to the LH) NPS injection, the peptide failed to increase cue-induced relapse. Of note is the fact that when NPS was given into the $\mathrm{LH}$, an increase of inactive lever response was observed, possibly reflecting general hyperarousal caused by NPS. On the other hand, it is unlikely that this locomotor-activating action of the peptide is responsible for the increase of alcohol seeking. In fact, the NPS effect at the active alcohol-paired lever remained significant even when it was re-analyzed using change-scores (active lever minus inactive lever presses).

The LH is the major source of Hcrt-1/Ox-A neurons in the brain, and it has already been demonstrated that these neurones co-express NPSR transcript and can be activated by NPS (Ciccocioppo et al, 2007; Niimi, 2006). Moreover, it has recently been recognized that Hcrt-1/Ox-A neurons within the LH play a key role in drug-seeking (Boutrel et al, 
2005; Dayas et al, 2008; Harris et al, 2005; Lawrence et al, 2006). Several NPS effects are similar to those of hypocretin, in that both these neuropeptides increase arousal, reduce sleep, and stimulate locomotor activity (Kotz et al, 2006; Xu et al, 2004). On the basis of these findings, we evaluated whether the NPS-induced increase of alcohol seeking requires activation of the Hcrt-1/Ox-A system. The finding that NPS effects to reinstate ethanol seeking were abolished by pre-treatment with the highly selective Hcrt-1/Ox-A antagonist SB-334867 provides support for this hypothesis. This finding suggests that stimulation of NPS receptors results in an activation of $\mathrm{Hcrt}-1 / \mathrm{Ox}-\mathrm{A}$ system activity that in turn, is responsible for potentiation of ethanol reinstatement. It is important to note that in our experiments we used SB-334867 at doses that did not modify alcohol reinstatement per se.

Several reports have shown that the Hcrt-1/Ox-A system plays a central role in the modulation of addictive behaviors for several classes of drugs of abuse including ethanol, cocaine and opiates and thus based on these findings, Hcrt1/Ox-A antagonism has been proposed as a possible pharmacological strategy for the treatment of drug addiction (Boutrel et al, 2005; Dayas et al, 2008; Harris et al, 2005; Lawrence et al, 2006). The results of this study not only provide additional evidence for the significance of the Hcrt$1 / \mathrm{Ox}-\mathrm{A}$ system in ethanol seeking and relapse, but also indicate for the first time that the NPS system has an important role as an upstream modulator of the Hcrt-1/OxA system. In addition, this study revealed some important differences between the effects of NPS and Hcrt-1/Ox-A on addictive behavior. For example, $\mathrm{LH}$ activation of the Hcrt$1 / \mathrm{Ox}-\mathrm{A}$ receptor system appears to be linked to the expression of conditioned ethanol seeking (Dayas et al, 2008; Lawrence et al, 2006) and also to have effects on alcohol consumption. Of note in this regard are studies that have shown that central infusion of Hcrt-1/Ox-A increases ethanol drinking in the rat (Schneider et al, 2007), whereas administration of the Hcrt-1/Ox-A antagonist SB-334867 reduces alcohol self-administration (Lawrence et al, 2006). In contrast, in our study we observed that the effects of NPS on addictive behavior differ from those of Hcrt-1/Ox-A; the former, in fact, specifically increases cue-induced reinstatement without affecting ethanol self-administration. At present, very little is known about the physio-pharmacology of the brain NPS system, a dearth that limits our ability to clarify the link between NPS and Hcrt-1/Ox-A neurotransmissions. Future studies aimed at systematically evaluating this relationship will certainly contribute to shedding new light on the neurobiology of drug addiction.

In conclusion, our results demonstrate for the first time that activation of NPSR in the LH increases relapse to alcohol seeking elicited by environmental conditioning factors. This effect is selective and is mediated by activation of $\mathrm{LH}$ hypocretin neurones. Based on the present findings, we also predict that antagonism at NPS receptors could represent a novel pharmacological approach to alcohol relapse treatment.

\section{ACKNOWLEDGEMENTS}

Supported by Grant PRIN 2006 to (RC). We thank Alfredo Fiorelli and Marino Cucculelli, for expert technical assistance, and Sheila Beatty for linguistic revision of the paper.

\section{DISCLOSURE/CONFLICT OF INTEREST}

The authors declare no conflict of interest.

\section{REFERENCES}

Adinoff B, Junghanns K, Kiefer F, Krishnan-Sarin S (2005). Suppression of the HPA axis stress-response: implications for relapse. Alcohol Clin Exp Res 29: 1351-1355.

Badia-Elder NE, Henderson AN, Bertholomey ML, Dodge NC, Stewart RB (2008). The effects of neuropeptide S on ethanol drinking and other related behaviors in alcohol-preferring and -nonpreferring rats. Alcohol Clin Exp Res 32: 1380-1387.

Beck B, Fernette B, Stricker-Krongrad A (2005). Peptide S is a novel potent inhibitor of voluntary and fast-induced food intake in rats. Biochem Biophys Res Commun 332: 859-865.

Boutrel B, Kenny PJ, Specio SE, Martin-Fardon R, Markou A, Koob GF et al (2005). Role for hypocretin in mediating stress-induced reinstatement of cocaine-seeking behavior. Proc Natl Acad Sci USA 102: 19168-19173.

Ciccocioppo R, Angeletti S, Weiss F (2001). Long-lasting resistance to extinction of response reinstatement induced by ethanolrelated stimuli: role of genetic ethanol preference. Alcohol Clin Exp Res 25: 1414-1419.

Ciccocioppo R, Kallupi M, Cannella N, Braconi S, Stopponi S, Economidou D (2007). Neuropeptide S system activation facilitate conditioned reinstatement of cocaine seeking in the rat. Society for Neuroscience 2007, San Diego, pp. 271.18/Z1. Ref Type: Abstract.

Cippitelli A, Cannella N, Braconi S, Duranti A, Tontini A, Bilbao A et al (2008). Increase of brain endocannabinoid anandamide levels by FAAH inhibition and alcohol abuse behaviours in the rat. Psychopharmacology 198: 449-460.

Cleck JN, Blendy JA (2008). Making a bad thing worse: adverse effects of stress on drug addiction. J Clin Invest 118: 454-461.

Dayas CV, McGranahan TM, Martin-Fardon R, Weiss F (2008). Stimuli linked to ethanol availability activate hypothalamic CART and orexin neurons in a reinstatement model of relapse. Biol Psychiatry 63: 152-157.

de Lecea L, Kilduff TS, Peyron C, Gao XB, Foye PE, Danielson PE et al (1998). The hypocretins: hypothalamus-specific peptides with neuroexcitatory activity. Proc Natl Acad Sci USA 95: 322-327.

de Lecea L, Sutcliffe JG (2005). The hypocretins and sleep. FEBS $J$ 272: 5675-5688.

Economidou D, Mattioli L, Cifani C, Perfumi M, Massi M, Cuomo $\mathrm{V}$ et al (2006). Effect of the cannabinoid CB1 receptor antagonist SR-141716A on ethanol self-administration and ethanol-seeking behaviour in rats. Psychopharmacology (Berl) 183: 394-403.

Hagan JJ, Leslie RA, Patel S, Evans ML, Wattam TA, Holmes S et al (1999). Orexin A activates locus coeruleus cell firing and increases arousal in the rat. Proc Natl Acad Sci USA 96: 10911-10916.

Harris GC, Wimmer M, Aston-Jones G (2005). A role for lateral hypothalamic orexin neurons in reward seeking. Nature 437: 556-559.

Heilig M, Koob GF (2007). A key role for corticotropin-releasing factor in alcohol dependence. Trends Neurosci 30: 399-406.

Jungling K, Seidenbecher T, Sosulina L, Lesting J, Sangha S, Clark SD et al (2008). Neuropeptide S-mediated control of fear expression and extinction: role of intercalated GABAergic neurons in the amygdala. Neuron 59: 298-310. 
Katner SN, Magalong JG, Weiss F (1999). Reinstatement of alcoholseeking behavior by drug-associated discriminative stimuli after prolonged extinction in the rat. Neuropsychopharmacology 20: 471-479.

Kotz CM, Wang C, Teske JA, Thorpe AJ, Novak CM, Kiwaki K et al (2006). Orexin A mediation of time spent moving in rats: neural mechanisms. Neuroscience 142: 29-36.

Lawrence AJ, Cowen MS, Yang HJ, Chen F, Oldfield B (2006). The orexin system regulates alcohol-seeking in rats. $\mathrm{Br}$ J Pharmacol 148: $752-759$.

Le A, Shaham Y (2002). Neurobiology of relapse to alcohol in rats. Pharmacol Ther 94: 137-156.

Le AD, Harding S, Juzytsch W, Funk D, Shaham Y (2005). Role of alpha-2 adrenoceptors in stress-induced reinstatement of alcohol seeking and alcohol self-administration in rats. Psychopharmacology 179: 366-373.

Niimi M (2006). Centrally administered neuropeptide $S$ activates orexin-containing neurons in the hypothalamus and stimulates feeding in rats. Endocrine 30: 75-79.

Reinscheid RK, Xu YL, Civelli O (2005). Neuropeptide S: a new player in the modulation of arousal and anxiety. Mol Interv 5: 42-46.

Sakurai T, Amemiya A, Ishii M, Matsuzaki I, Chemelli RM, Tanaka $\mathrm{H}$ et al (1998). Orexins and orexin receptors: a family of hypothalamic neuropeptides and $\mathrm{G}$ protein-coupled receptors that regulate feeding behavior. Cell 92: 573-585.

Schneider ER, Rada P, Darby RD, Leibowitz SF, Hoebel BG (2007). Orexigenic peptides and alcohol intake: differential effects of orexin, galanin, and ghrelin. Alcohol Clin Exp Res 31: 1858-1865.

Smith KL, Patterson M, Dhillo WS, Patel SR, Semjonous NM, Gardiner JV et al (2006). Neuropeptide S stimulates the hypothalamo-pituitary-adrenal axis and inhibits food intake. Endocrinology 147: 3510-3518.

Stewart RB, Gatto GJ, Lumeng L, Li TK, Murphy JM (1993). Comparison of alcohol-preferring $(\mathrm{P})$ and nonpreferring (NP) rats on tests of anxiety and for the anxiolytic effects of ethanol. Alcohol 10: 1-10.

Vitale G, Filaferro M, Ruggieri V, Pennella S, Frigeri C, Rizzi A et al (2008). Anxiolytic-like effect of neuropeptide $S$ in the rat defensive burying. Peptides 12: 2286-2291.

Xu YL, Gall CM, Jackson VR, Civelli O, Reinscheid RK (2007). Distribution of neuropeptide $S$ receptor mRNA and neurochemical characteristics of neuropeptide S-expressing neurons in the rat brain. J Comp Neurol 500: 84-102.

Xu YL, Reinscheid RK, Huitron-Resendiz S, Clark SD, Wang ZW, Lin SH et al (2004). Neuropeptide S: a neuropeptide promoting arousal and anxiolytic-like effects. Neuron 43: 487-497. 